\title{
Objetos Virtuales de Aprendizaje en la enseñanza de las Ciencias Sociales
}

Elizabeth Arenas Gaona*

Artículo de reflexión

Fecha de Recepción: 20 mayo 2018.

Fecha de Aprobación: 1 septiembre 2018.

\section{Resumen}

La presente ponencia presenta los avances del proyecto de investigación intitulado: "Objetos virtuales de aprendizaje (OVA) en la enseñanza de las Ciencias Sociales", adscrito a la Maestría en Educación con énfasis en profundización de la Universidad Pedagógica y Tecnológica de Colombia, cuyo propósito fundamental es evidenciar la aplicación de la estrategia "Sociales Android" diseñada por la docente investigadora a partir de una aplicación Android, con el fin de transformar y enriquecer las prácticas docentes en el área de Ciencias Sociales.

El proyecto se desarrolla en el municipio de Jenesano (Boyacá), en la Institución Educativa Técnico Comercial, con estudiantes de grado sexto que cuentan con edades entre los 11 y los 15 años. La metodología se inscribe dentro del tipo de investigación cualitativa y el enfoque es el de la Investigación Acción Educativa, las técnicas de investigación usadas fueron la exploración documental, la observación participante y el grupo focal, por su parte los instrumentos fueron el plan de área, los planeadores de clase, fichas de registro, el diario de campo y la entrevista semiestructurada.

En cuanto a los resultados parciales del proyecto y teniendo en cuenta el tipo de evaluación que se usó a lo largo
* Institución Educativa Técnico Comercial de Jenesano - Boyacá Colombia lizuptc@gmail.com 
de la investigación, se puede mostrar que los estudiantes mejoraron sus procesos cognitivos, procedimentales y actitudinales y la docente mejoró su proceso pedagógico con el uso del OVA. Como conclusión los OVA abren posibilidades infinitas para las aulas de clase y para la formación de los docentes, por lo cual se hace necesario que desde las universidades se implementen currículos que los tengan en cuenta.

Palabras Clave: Entornos Virtuales de Aprendizaje, Objeto Virtual de Aprendizaje, Constructivismo, Competencias TIC para el desarrollo profesional docente y Ciencias Sociales. 


\section{Introducción}

El trabajo de investigación se llevó a cabo en el Municipio de Jenesano (Boyacá), ubicado en una de las estribaciones de la cordillera oriental en la provincia de Márquez, a treinta kilómetros de la ciudad de Tunja. Allí se encuentra la Institución Educativa Técnico Comercial de Jenesano, Establecimiento educativo de carácter público y que cuenta con quince sedes rurales, una urbana de primaria y la sede central que ofrece los niveles de educación secundaria, media y técnica en las modalidades de comercio y sistemas, está ubicada en el barrio Naranjos y cuenta con 657 estudiantes (Institución Educativa Técnico Comercial de Jenesano, 2017).

La muestra escogida para el desarrollo del proyecto corresponde al grado 6-2, que cuenta con 32 estudiantes, 17 niñas y 15 niños con edades entre los $11 \mathrm{y}$ los 14 años. En este curso 13 jóvenes viven en la zona urbana y 19 en la zona rural del municipio, tras una fase de observación se pudo inferir que los estudiantes que viven en la cabecera urbana del municipio tienen mayor facilidad de expresión y conectividad al contar con mayores posibilidades de acceso a computadores, tabletas y celulares que los estudiantes que viven en la zona rural que por el contrario tienen limitaciones de conectividad y tienden a ser menos expresivos. Sin embargo de acuerdo a la observación participante que se llevó a cabo, se pudo establecer con certeza que todos los estudiantes tienen habilidades y capacidades en el manejo de las tabletas electrónicas.
En este orden de ideas la problemática a tratar en la presente ponencia se plantea desde las dificultades encontradas en las prácticas pedagógicas del área de Ciencias Sociales en la Institución Educativa Técnico Comercial de Jenesano, ya que a través de ejercicios de caracterización por medio de fichas de registro y exploración documental, se pudo constatar que las prácticas actuales distan de las propuestas en el modelo pedagógico constructivista establecido en el PEI de la Institución Educativa, que dice:

Los pedagogos de la Escuela Nueva enfatizaron en el "principio de la actividad", en el sentido que es haciendo y experimentando como el niño aprende, es desde la propia actividad vital del niño como este se desarrolla; partiendo de sus intereses y necesidades es como el niño se autoconstruye y se convierte en protagonista y eje de todo el proceso educativo (Institución Educativa Técnico Comercial de Jenesano, 2017)

Por otra parte tampoco se potencian al máximo las capacidades y aptitudes de los estudiantes, al no desarrollarse un trabajo personalizado en el aula de clase.

De esta situación nace la propuesta pedagógica de crear un OVA (aplicación móvil con contenidos programáticos del área, para primer y segundo periodo lectivo) con el fin de analizar hasta qué punto se pueden revitalizar y dinamizar las clases en las aulas y atraer la atención de los estudiantes, profundizando sus dimensiones cognitiva, afectiva y comunicativa. Al
Los pedagogos de la Escuela Nueva enfatizaron en el "principio de la actividad", en el sentido que es haciendo y experimentando como el niño aprende, es desde la propia actividad vital del niño como este se desarrolla; partiendo de sus intereses y necesidades es como el niño se autoconstruye y se convierte en protagonista y eje de todo el proceso educativo 
Dentro de la dimensión cognitiva del aprendizaje se concede especial relevancia al papel de variables como las aptitudes o procesos cognitivos, los conocimientos previos, los estilos cognitivos y de aprendizaje y, especialmente,

las estrategias generales y específicas de aprendizaje respecto de la dimensión cognitiva Núñez Pérez et al. (1998) afirma:

Dentro de la dimensión cognitiva del aprendizaje se concede especial relevancia al papel de variables como las aptitudes o procesos cognitivos, los conocimientos previos, los estilos cognitivos y de aprendizaje y, especialmente, las estrategias generales y específicas de aprendizaje... (p.98).

Si bien establecer los conocimientos previos es parte de las prácticas cotidianas en el aula de clase, la determinación de estilos cognitivos no complementa este ejercicio, por lo que para el proceso investigativo se tuvieron en cuenta las variables cognitivas señaladas por los autores, observándose cambios significativos en los niños y niñas de grado sexto que participaron en el desarrollo de la propuesta, ya que enriquecieron su proceso de aprendizaje con conocimientos previos no solo de la asignatura sino en el manejo de TIC; por otra parte la investigación demuestra que los ritmos diferenciados de aprendizaje, y el uso tabletas electrónicas en el aula de clase potenció de manera positiva la adquisición del conocimiento de los niños y niñas.

Por otro lado, la intención de abordar la dimensión afectiva se encuentra directamente relacionada con las interacciones sociales que en la escuela se presentan a diario y que influyen directamente en la enseñanza y aprendizaje. La observación del lenguaje no verbal y verbal permitió determinar un aumento en el nivel de interés y una mayor participación en las clases utilizando recursos electrónicos como herramientas de mediación para el aprendizaje de las Ciencias Sociales a través de la generación de vínculos afectivos, de comprensión, aceptación y apoyo. $\mathrm{Al}$ respecto se trabajó con base en las ideas planteadas por Hernández y Sánchez (2008) quienes afirman:

Uno de esos aspectos relevantes que transversalizan todas las etapas del ciclo de vida es el vínculo afectivo, el cual tiene su expresión en la posibilidad de construir lazos o uniones de cariño, comprensión, tolerancia, aceptación, empatía, apoyo, comunicación, que se dan principalmente entre las relaciones que establecen los padres con sus hijos y en su defecto los niños con sus cuidadores (p.56).

Como un componente transversal asociado a los proyectos de competencias ciudadanas, cátedra para la paz y convivencia, se trabaja con el desarrollo de la dimensión comunicativa de los estudiantes por medio de la cual ellos expresan sentimientos emociones, necesidades y pensamientos, que son fundamentales en la Investigación Acción Educativa. La búsqueda de nuevas formas de expresión ha permitido generar estudiantes con mayor expresión verbal, gestual y un mayor autoconocimiento de sus propias capacidades y autonomía al explorar por sí mismos las posibilidades de la aplicación desarrollada acorde al ritmo de cada uno.

De igual manera la propuesta busca afianzar las competencias en TIC, en los ámbitos pedagógico, tecnológico, 
investigativo y comunicativo para el mejoramiento del proceso de enseñanza y aprendizaje de las Ciencias Sociales en la básica secundaria.

La ruta seguida para llevar a cabo el desarrollo de la investigación, parte del análisis del contexto con un enfoque cualitativo que conduce a un mejor reconocimiento del ambiente escolar en el que el investigador está inmerso, lo cual implica que no sea un actor pasivo o que simplemente observa y describe fenómenos, sino que también es parte del problema y por ende de su solución. Por lo anterior, es indispensable descentrarse de la realidad educativa para poder analizarla, reconocer la problemáticas que afectan la práctica pedagógica, y la indagación de las estrategias pertinentes para enriquecer de experiencias significativas dirigidas tanto para el docente, como a los estudiantes, a través de la validación y la sistematización de vivencias, de esta manera se fortalecen las interacciones y las relaciones interpersonales, ayudando esto al mejoramiento del clima escolar.

Este proyecto se inscribe dentro del enfoque de investigación social cualitativa, para dar cuenta de las problemáticas del fenómeno educativo, al respecto se tienen en cuenta los planteamientos de Hernández, Fernández y Baptista (2010) cuando afirman: "(...) la investigación cualitativa se enfoca a comprender y profundizar los fenómenos, explorándolos desde la perspectiva de los participantes en un ambiente natural y en relación con el contexto" (p.406). Por lo cual, la tecnología adecuada considerada para desarrollar como proyecto de investigación, es el Objeto Virtual de Aprendizaje (OVA) ya que por su versatilidad permite enriquecer las prácticas pedagógicas en las Ciencias Sociales, tomando en cuenta la subjetividad, las opiniones, expresiones y sentimientos de los estudiantes buscando transformar la práctica educativa donde el papel del docente; es el de orientador del proceso pedagógico generando un rol más activo para el estudiante como constructor de su propio conocimiento.

\section{Problematizando la Escuela}

En la actualidad la escuela se enfrenta a grandes dificultades en cuanto al uso de nuevas herramientas metodológicas por parte de los docentes en su interacción con los estudiantes y el conocimiento, sin embargo la formación docente generalmente está desfasada con relación a los rápidos avances de las TIC y sus posibilidades en el aula de clase, que en muchas ocasiones ni siquiera son implementadas, no se tiene la capacidad tecnológica instalada o la actualización docente requerida; por lo tanto los esfuerzos de la pedagogía tradicional no son suficientes para atraer el interés de los estudiantes ya que las prácticas docentes tradicionales no pueden formar a los estudiantes del siglo XXI, al respecto Serres (2012) afirma: "Él o ella no tiene el mismo cuerpo, la misma esperanza de vida, no se comunica más de la misma manera, no percibe ya el mismo mundo, no vive en la misma naturaleza, ya no habita 
el mismo espacio"(p.8). Este cambio contextual debe ser el centro de interés tanto del docente como como del estudiante, lo cual conduce a repensar la Escuela, la educación y los procesos de enseñanza aprendiendo a usar las nuevas herramientas tecnológicas, de las que se valen los niños, niñas y adolescentes para aprender, con el fin de acercarse de forma más asertiva a ellos y de esta manera poder iniciar un proceso de realimentación que conduzca finalmente al logro de las metas del proceso de enseñanza y aprendizaje.

Las prácticas pedagógicas tienden a ser impuestas desde el docente sin tener en cuenta las necesidades e intereses de los estudiantes y su contexto según la mentalidad de la pedagogía tradicional, en la cual el docente es quien enseña y el estudiante es quien aprende un conocimiento previamente establecido como contenido, sin mayor problematización, esta situación redunda en el uso descontextualizado de un único texto escolar, el cual no ha tenido la intervención del docente o del estudiante y no tiene en cuenta al medio en el que viven los niños y sus intereses. Por el contrario el paradigma actual propone que sea la construcción participativa y colaborativa entre el docente y el estudiante, el resultado de la interacción y problematización de un hecho, fenómeno u objeto de aprendizaje.

En cuanto a las TIC los avances tecnológicos requieren de una actualización continua, de una selección cuidadosa de textos, imágenes, videos, sonidos que genera una relación interactiva con el estudiante y que por avance de la información y la tecnología requiere ser actualizado cada 18 meses por lo cual es necesario justificar su uso. Con respecto al uso de textos escolares en medio físico, los OVA permiten este proceso de actualización continua $\mathrm{y}$ flexible ahorrando costos materiales, ambientales y de manejo de espacios.

En el área de Ciencias Sociales haberse pensado el problema implicó una experiencia directa con los estudiantes y por ende las bases de la disciplina buscan ser reconceptualizadas y coconstruidas a la luz de las experiencias pedagógicas diseñadas e implementadas. Por ejemplo Esterberg (como se citó en Hernández et al., 2010) afirma:

Bajo la búsqueda cualitativa, en lugar de iniciar con una teoría particular y luego "voltear" al mundo empírico para confirmar si ésta es apoyada por los hechos, el investigador comienza examinando el mundo social y en este proceso desarrolla una teoría coherente con los datos, de acuerdo con lo que observa, frecuentemente denominada teoría fundamentada (p.9).

Otra de las razones por las cuales el enfoque cualitativo es pertinente para el desarrollo de la investigación, es porque como lo dice Esterberg (2002) no se pretende tomar una teoría ampliamente conocida y verificada para ponerla a prueba en las aulas de clase. En la presente investigación se buscó comprender y analizar situaciones educativas desde las Ciencias Sociales que puedan considerarse fuente de 
información para finalmente hacer una posible teorización, que pueda ser útil a las prácticas docentes y a futuras investigaciones.

El enfoque cualitativo en sus diferentes intencionalidades busca diseñar rutas, caminos y estrategias particulares, por ello, el tipo de investigación desarrollado correspondió a ejercicios de investigación propios de la educación para lo cual se tomó la Investigación Acción Educativa en la que el docente indaga, toma parte activa del proceso, relacionando problemas y experiencias de la investigación en la Institución Educativa que se detectaron en el aula. Al respecto Elliot (2000) afirma:

La investigación-acción se relaciona con los problemas prácticos cotidianos experimentados por los profesores, en vez de con los "problemas teóricos" definidos por los investigadores puros en el entorno de una disciplina del saber. Puede ser desarrollada por los mismos profesores o por alguien a quien ellos se lo encarguen (p.5).

De esta manera la IAE es la herramienta metodológica seleccionada para abordar los problemas de la práctica pedagógica en la Institución Educativa y proponer estrategias de solución incluyendo a los estudiantes del grado sexto en el proceso para que sea más significativo y contextualizado con las problemáticas, temas y contenidos.

(...) las situaciones a las que se enfrentan los profesores en la actualidad cambian constantemente, de modo que los hábitos y rutinas docentes establecidos con antelación dejan de ser útiles. Esas situaciones requieren el continuo desarrollo de "teorías-en-la-acción" mediante la investigación-acción, con la condición de evitar las pautas docentes establecidas. Con el fin de desarrollar las nuevas técnicas, los profesores deben disponerse a soportar la experiencia de ser "destecnificados” (Elliot, 2000 p.16).

Es completamente necesario encarar la situación dela Educación en el momento actual, que requiere generar conciencia y también un cambio de mentalidad que implica pensar el acto educativo en el área de las Ciencias Sociales desde la competencia investigativa, tecnológica, comunicativa y de gestión problematizando todas las situaciones en el trabajo dentro y fuera del aula. De esta manera y con el fin de especificar la ruta usada para el desarrollo del proyecto de investigación, el primer paso caracterizó el contexto de las prácticas pedagógicas desarrolladas con los estudiantes de grado sexto, para lo cual se usó la técnica de exploración de documentos, teniendo en cuenta el plan que maneja el área de Ciencias Sociales, los planeadores de clase en donde los docentes consignan las actividades específicas que realizan en su práctica cotidiana y las fichas de registro.

Una fuente muy valiosa de datos cualitativos son los documentos, materiales y artefactos diversos. Nos pueden ayudar a entender el fenómeno central de estudio. Prácticamente la mayoría de las personas, grupos, organizaciones, comunidades y sociedades los producen y narran, o delinean sus
La investigación-acción se relaciona con los problemas prácticos cotidianos experimentados por los profesores, en vez de con los "problemas teóricos" definidos por los investigadores puros en el entorno de una disciplina del saber. Puede ser desarrollada por los mismos profesores o por alguien a quien ellos se lo encarguen 
Desde el marco de interpretación constructivista de la enseñanza y el aprendizaje, la evaluación es una actividad que debe realizarse tomando en cuenta no solo el aprendizaje de los alumnos, sino también las actividades de enseñanza que realiza el docente y su relación con dichos aprendizajes.

En este caso, entonces, las acciones evaluativas se encaminarán a reflexionar, interpretar y mejorar dicho proceso desde el interior del mismo (evaluación para y en el proceso enseñanza y aprendizaje historias y estatus actuales. Le sirven al investigador para conocer los antecedentes de un ambiente, las experiencias, vivencias o situaciones y su funcionamiento cotidiano. (Hernández, et al., 2010, p.433).

La exploración de documentos se hizo con el fin de analizar y reflexionar acerca del proceso de enseñanza de las Ciencias Sociales en la Institución Educativa, tomando como referencia las estrategias pedagógicas, los recursos, la evaluación y observaciones de los docentes para luego replantear las prácticas educativas que han perdurado por muchos años y que han sido nocivas para el proceso de aprendizaje de los estudiantes, entre ellos la clase magistral y recursos como el video y el libro de texto.

El segundo paso para la ejecución del proyecto de aula fue el diseño y puesta en práctica de una aplicación móvil para tabletas electrónicas, con contenidos programáticos específicos del área de Ciencias Sociales para el primer y segundo periodo, con los estudiantes de grado sexto, que ha sido diseñada por la docente investigadora y sobre la cual se analizan y validan los errores y se complementa la Aplicación con las sugerencias de los estudiantes.

Como técnica de investigación se usó la observación participante que requiere que el investigador tenga todos sus sentidos enfocados en aquello que es relevante para su proyecto (Hernández, et al., 2010), para esta técnica el instrumento correspondiente fue el diario de campo, en el cual se registraron y sistematizaron datos, experiencias, procesos e información importantes que se llevaron a cabo en el aula con los estudiantes que hicieron parte de la muestra y que luego fueron analizados desde autores que soportaron las categorías que en ellos se pudieron vislumbrar.

El tercer paso para la culminación del proyecto de investigación se encaminará a la valoración del desempeño de los estudiantes en el área de Ciencias Sociales con el uso del aplicativo móvil, por medio de la evaluación desde el aprendizaje significativo de los procesos procedimentales, cognitivos y actitudinales. Al respecto Coll \& Martín, 1996 (como se citó en Díaz \& Hernández, 2010) afirman:

Desde el marco de interpretación constructivista de la enseñanza y el aprendizaje, la evaluación es una actividad que debe realizarse tomando en cuenta no solo el aprendizaje de los alumnos, sino también las actividades de enseñanza que realiza el docente y su relación con dichos aprendizajes. En este caso, entonces, las acciones evaluativas se encaminarán a reflexionar, interpretar y mejorar dicho proceso desde el interior del mismo (evaluación para y en el proceso enseñanza y aprendizaje (p.309).

En este orden de ideas y siguiendo con el curso de la ponencia se presenta la discusión en la que se confronta el punto de vista de la investigadora con algunos autores, de acuerdo a unas categorías y subcategorías de análisis que son el resultado del proceso 
metodológico del trabajo realizado en el aula; teniendo en cuenta a Zemelman (2017) cuando habla del pensamiento teórico y el pensamiento epistémico, mencionando el desfase que se produce frecuentemente entre teoría y práctica a la hora de hacer una investigación, ya que los conceptos se construyen a un ritmo más lento que la realidad que queremos estudiar, conllevando a una falsación del conocimiento, por lo cual es pertinente tener una postura propia frente a la teoría, de esta manera se deja por sentado que el análisis de las categorías va a estar cruzado por una visión ajustada al contexto.

Entornos Virtuales de Aprendizaje (EVA)

Las aulas son espacios ideales como "Entornos Virtuales de Aprendizaje" lo cual implica una transformación a través de la problematización de los roles, de las actividades a desarrollar y de los conocimientos y saberes que deben interactuar(Pulido, Najar \& Guesguan, 2016). Para ello se parte de su definición como espacios que:

- ...Se adaptan a las características y necesidades del usuario. Para ello, disponen de diferentes roles en relación a la actividad que realizan en el EVA: administrador, profesor, tutor y estudiante. Los privilegios de acceso están personalizados y dependen del rol del usuario. De modo que, el EVA debe de adaptarse a las necesidades del usuario particular.

- Posibilitan la comunicación e interacción entre los estudiantes y el profesor-tutor.
- Presenta diferentes tipos de actividades que pueden ser implementadas en un curso.

- Incorporan recursos para el seguimiento y evaluación de los estudiantes (Belloch, s.f.p.2).

Para el caso particular, estos EVA fueron propicios para la creación de la Aplicación Móvil que puede ser utilizada en diversos escenarios y no solamente en el aula de clase, ya que permiten tener en cuenta falencias y necesidades de la Institución Educativa como el escaso acceso a internet y el poco uso de las tabletas electrónicas para convertirlas en oportunidades para el proceso de enseñanza y aprendizaje de las Ciencias Sociales. Por otra parte permitieron que en las clases hubiese mayor comunicación entre los estudiantes y la docente por tratarse de un entorno por el que ellos y ellas se sintieron sumamente atraídos y alentados para aprender, asimismo contribuyeron significativamente al proceso de evaluación en el que los estudiantes aprendieron y aprehendieron conceptos propios de la asignatura.

Los Objetos Virtuales de Aprendizaje (OVA)

Una categoría emergente que se despliega de los Entornos virtuales de Aprendizaje EVA y que jugó un papel fundamental en la investigación fue la de los Objetos Virtuales de Aprendizaje, (OVA), los cuales define Botero (2014) como herramienta: 
Dentro de la gran variedad de definiciones que se pueden encontrar, se destaca que la mayoría conciben la OVA como una herramienta o recurso interactivo, el cual debe presentar unas características de accesibilidad, interactividad y portabilidad, para que cualquier persona pueda acceder a él de forma simultánea o individual, y que facilite realizar un aprendizaje colaborativo (p.19).

La construcción de una propuesta "Sociales Android"

Para el desarrollo de la investigación en el aula el OVA diseñado se denominó: "Sociales Android", que partió de la caracterización del contexto escolar de la Institución Educativa y fue pensada, diseñada y desarrollada para los niños y niñas de grado sexto; para lo cual se apoya en el manejo de la imagen: los dibujos, esquemas, videos, imágenes en movimiento y juegos acordes, por un lado con los estándares que maneja el Ministerio de Educación Nacional (2004) y por otro con las necesidades de los estudiantes y la docente, ya que es una plataforma de fácil accesibilidad, no requiere conexión a internet, es interactiva y se puede instalar en cualquier dispositivo móvil que soporte Android.

La aplicación cuenta con una estructura que parte de un menú de unidades, parejas, sopa de letras, crucigrama, ahorcado y contenido.

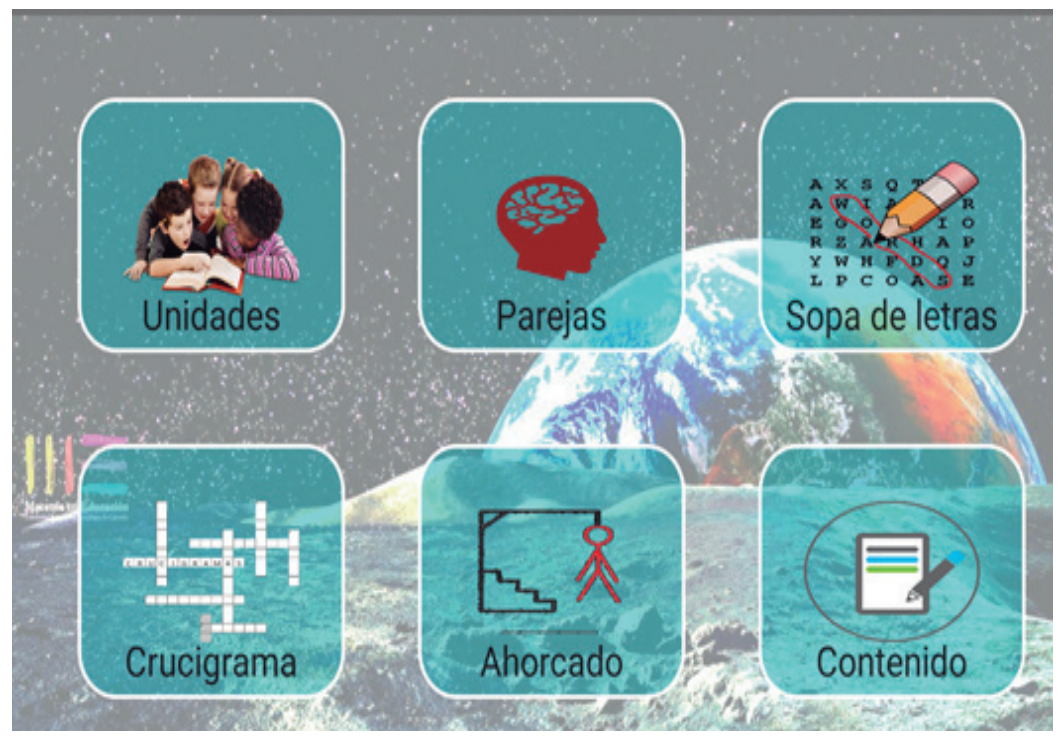

[Fotografía de Elizabeth Arenas]. (Tunja, 2017) Archivo personal 
Al tocar en contenido, aparecen cuatro unidades, cada una con sus sub temas.

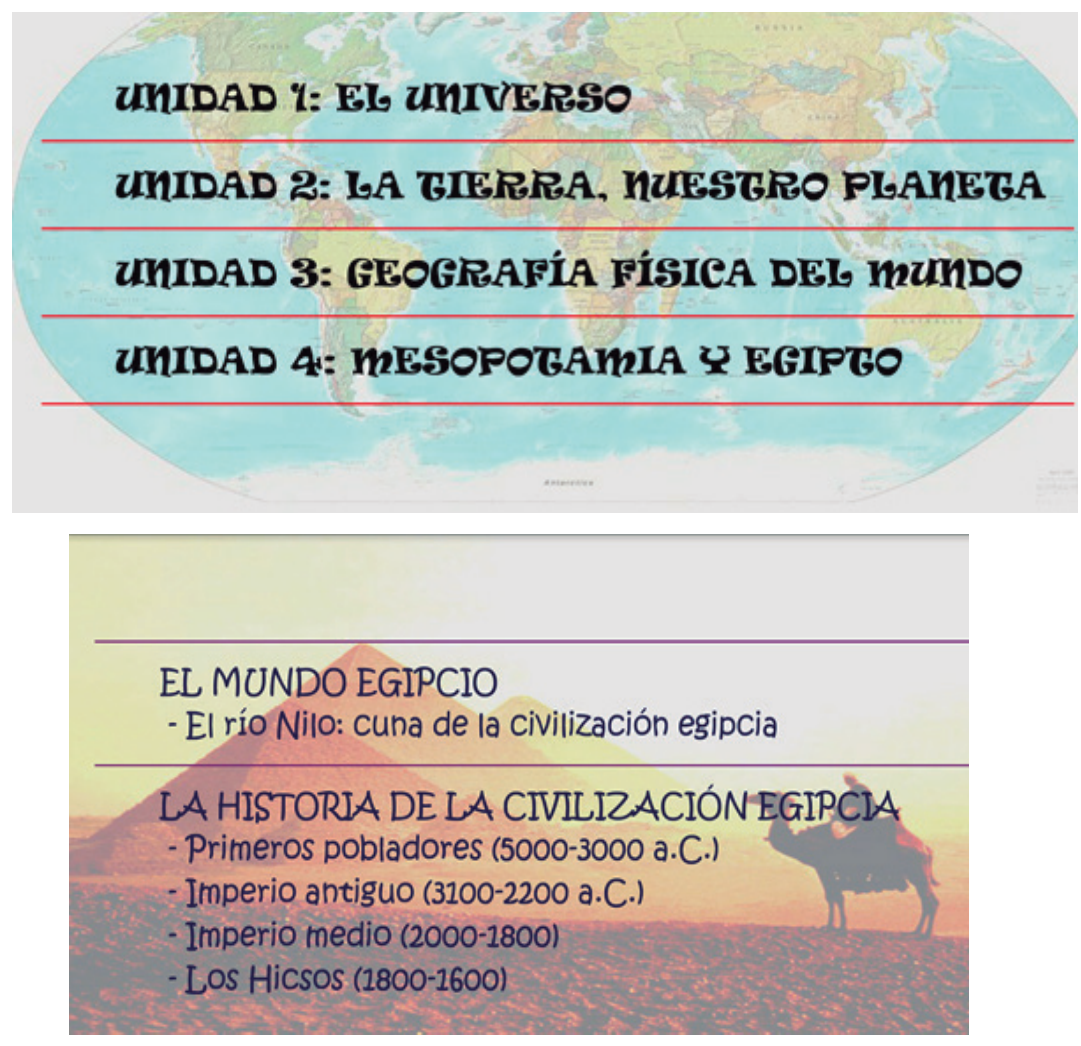

[Fotografías de Elizabeth Arenas]. (Tunja, 2017) Archivo personal

Al tocar en unidades se abre otro menú en el que se encuentra; Unidad 1, Unidad 2, Unidad 3 y Unidad 4

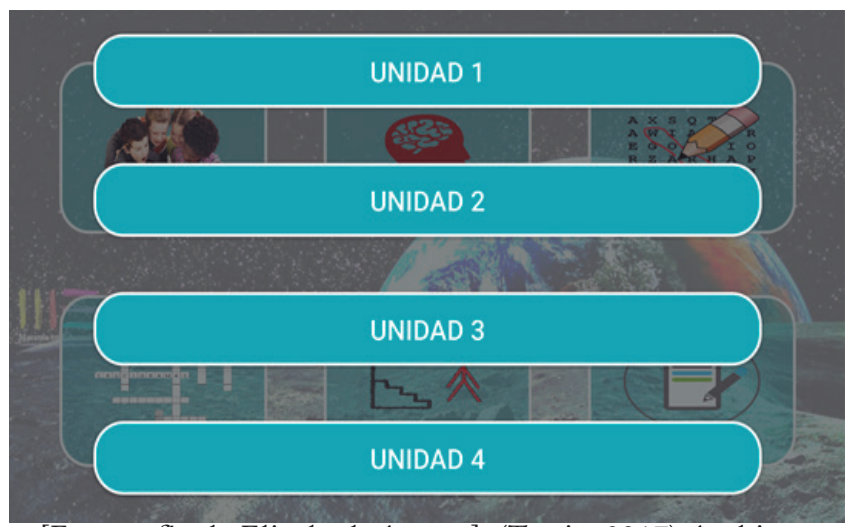

[Fotografía de Elizabeth Arenas]. (Tunja, 2017) Archivo personal 
De esta manera el estudiante puede empezar a explorar las unidades en las que se desarrolla el contenido de la asignatura.

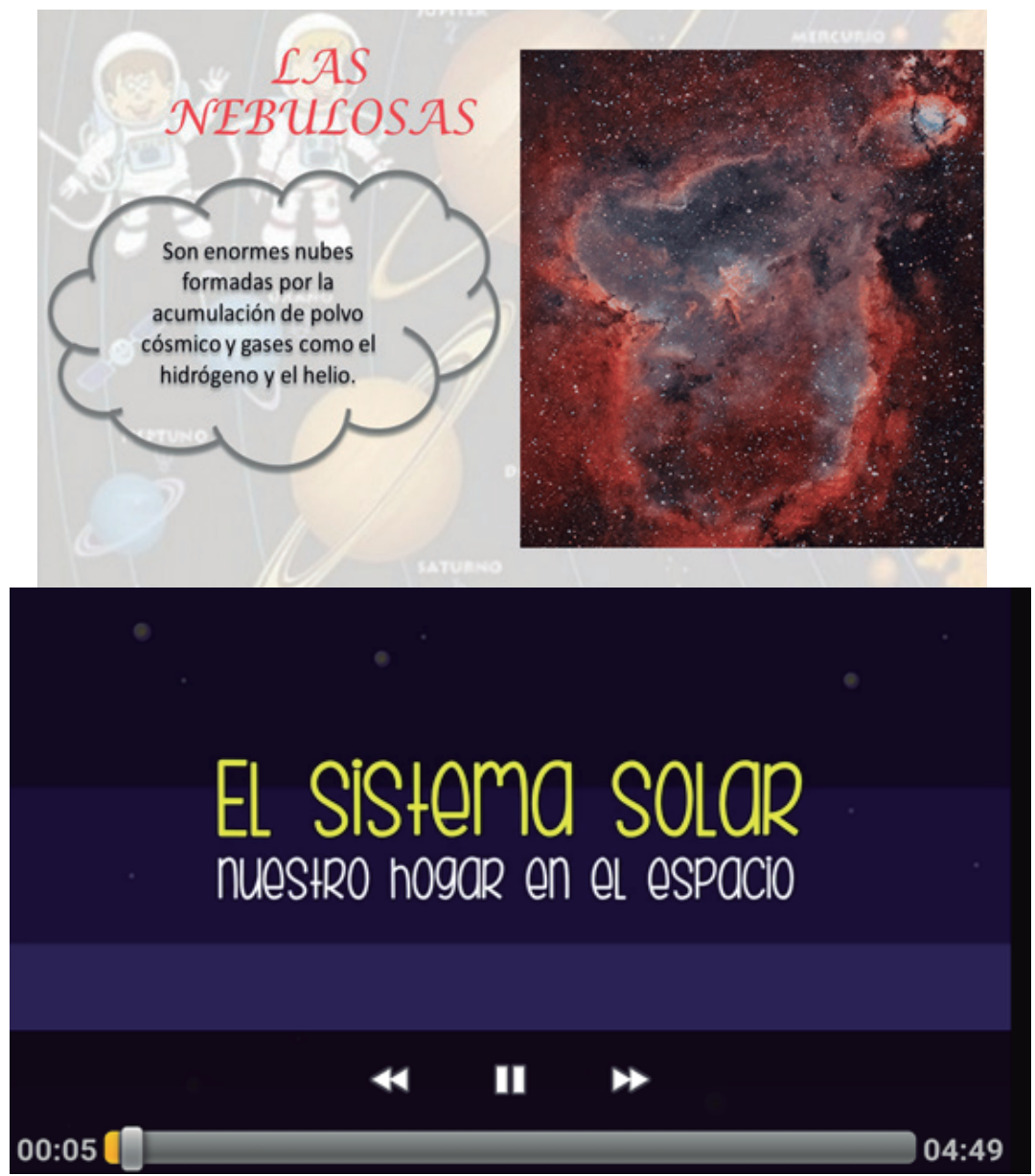

[Fotografías de Elizabeth Arenas]. (Tunja, 2017) Archivo personal

Al tocar en parejas el estudiante ingresa al primer juego en el que se debe escoger la unidad, acto seguido aparecen los niveles de dificultad, que son: aprender, muy fácil, fácil, medio, difícil y experto, cuando toca uno de estos niveles puede empezar a jugar descubriendo imágenes y palabras tratando de que se correspondan tomando en consideración que cuenta con un tiempo limitado. 

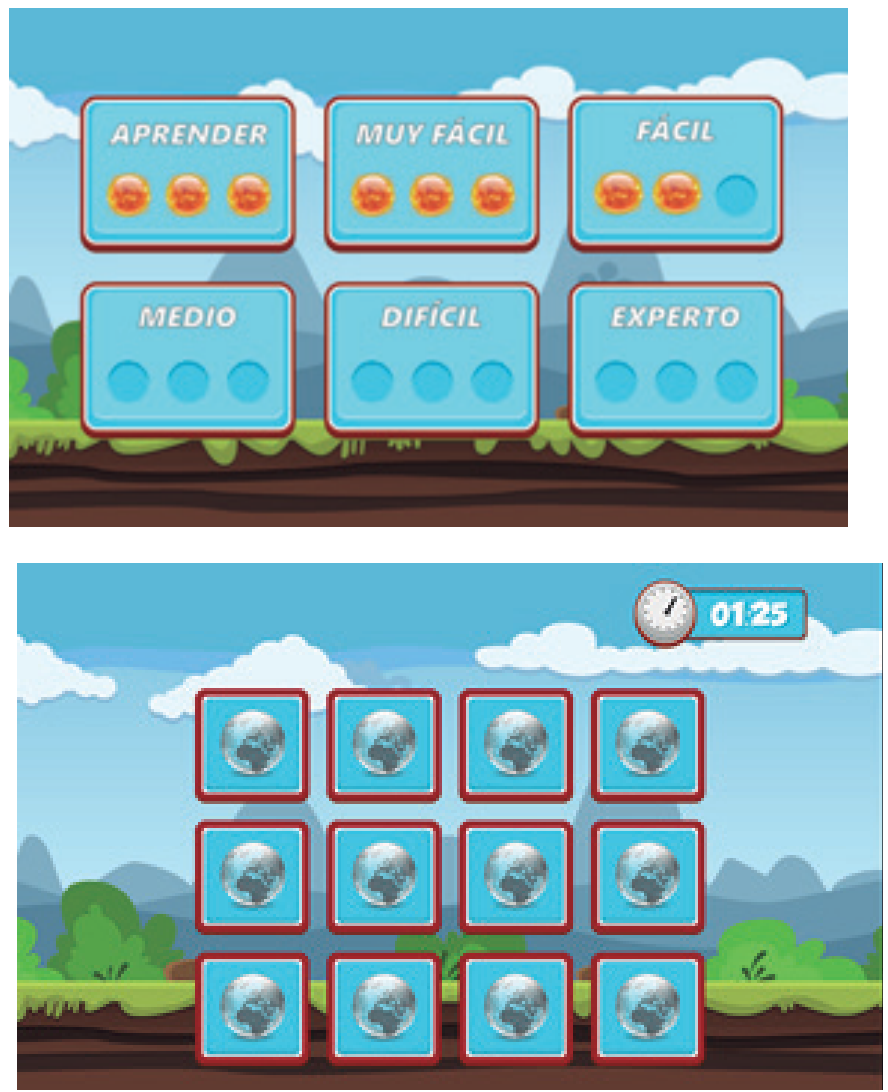

[Fotografías de Elizabeth Arenas]. (Tunja, 2017) Archivo personal

De igual manera cuando pulsan en sopas de letras y ahorcados, deben escoger la unidad, en el caso de los crucigramas estos se diferencian por sus nombres.

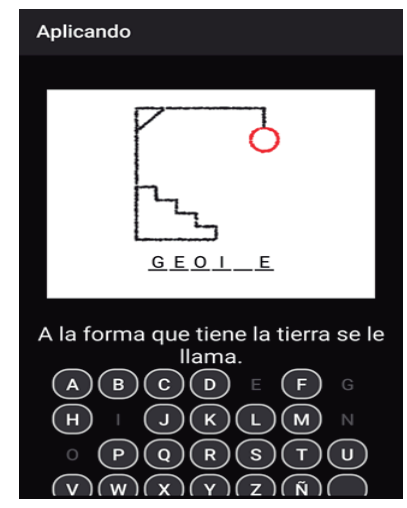

TGBASTEROIDE
ARINESPACIOA
OLUVEDACODR I
NBSPILIUORDC
OIEDMANOESON
INGMSILITTVE
RUUULDTADUG I
VCNTBETOCNLC
OERUPE R T T C
VVDAMETRAM R
UELOOLNTVGTA
AOCBIGBANGOD

[Fotografía de Elizabeth Arenas]. (Tunja, 2017) Archivo personal 


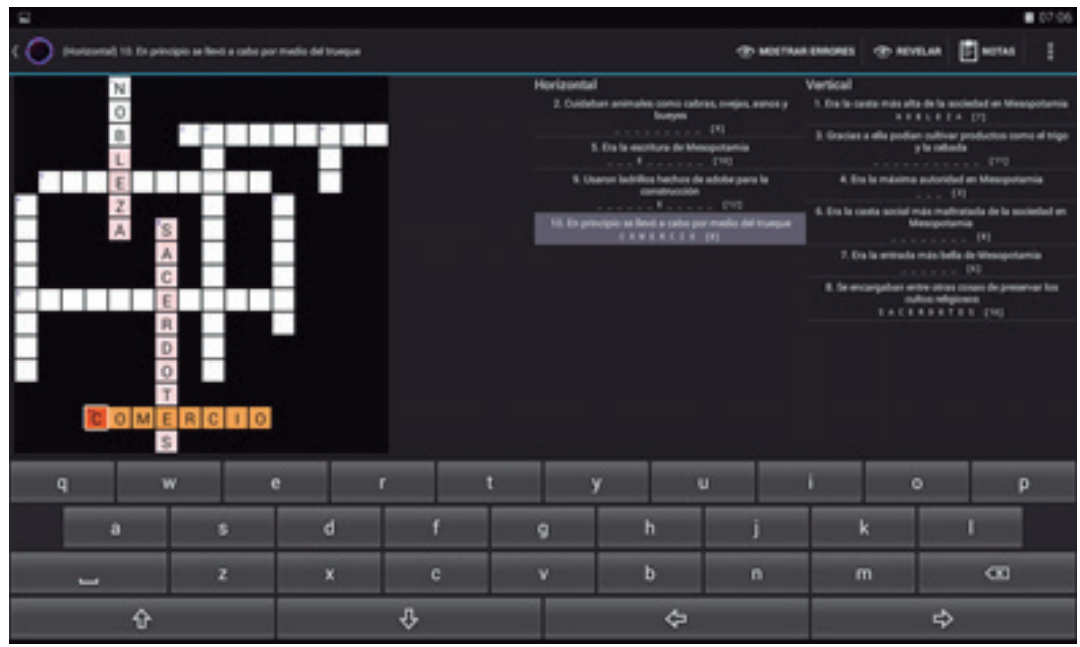

[Fotografía de Elizabeth Arenas]. (Tunja, 2017) Archivo personal

Dentro del contexto educativo, la competencia tecnológica se puede definir como la capacidad para seleccionar y utilizar de forma pertinente, responsable y eficiente una variedad de herramientas tecnológicas entendiendo los principios que las rigen, la forma de combinarlas y las licencias que las amparan"
Competencias TIC para el desarrollo profesional docente.

Por otro lado es importante considerar la necesidad de investigar el papel del docente y el desarrollo de competencias en tecnologías de información y comunicación, que hacen parte importante del proyecto de investigación porque es indudable que el rol del maestro se reconfigura dinámicamente en la actualidad y requiere de conocimientos básicos en estas tecnologías que los hagan competentes al lado de los estudiantes, que para el caso coparticipan del diseño y colaboran en algunos aspectos del desarrollo de la Aplicación, proceso que dio como resultado un alto grado de apropiación del resultado final y posibilitó un trabajo más significativo con los estudiantes, al respecto el Ministerio de Educación Nacional señala:
Dentro del contexto educativo, la competencia tecnológica se puede definir como la capacidad para seleccionar y utilizar de forma pertinente, responsable y eficiente una variedad de herramientas tecnológicas entendiendo los principios que las rigen, la forma de combinarlas y las licencias que las amparan" (Ministerio de Educación Nacional [MEN], 2013, p.31).

Cabe mencionar que aunque el MEN da estas disposiciones para las Instituciones Educativas públicas del país, muchas no cuentan con los recursos necesarios para poder cumplirlas, en el caso particular, aunque hay un punto vive digital $\mathrm{y}$ aunque la publicidad oficial dice: "Jenesano Vive digital", el colegio paga por el servicio de internet y de por sí es un servicio que presenta deficiencias en su conectividad y capacidad. Sin embargo el Ministerio dotó de 700 tabletas electrónicas al colegio en el año 2014, y 
capacitó a los docentes para incentivar su uso en las aulas de clase, teniendo esto en cuenta en la investigación se hace uso de ese mobiliario para activar el gran potencial que tienen los niños y niñas en el manejo de aparatos tecnológicos, direccionándolo hacia la adquisición del conocimiento de la asignatura de Ciencias Sociales, evitando que los equipos se dañen por desuso.

Estándares Básicos de Competencias en Ciencias Sociales

Parte importante al pensar en el desarrollo del proyecto de investigación era la articulación de los Estándares Básicos de Competencias en Ciencias Sociales (MEN 2004) en el OVA, ya que antes de la idea inicial se hizo una exploración por diferentes aplicaciones afines sin encontrar una que acogiera los contenidos de los dos primeros periodos lectivos del año escolar, es por ello que "Sociales Android" se convierte en una plataforma digital única porque incorpora los siguientes estándares básicos de competencias que exige el Ministerio de Educación Nacional en Ciencias Sociales, en cuanto a relaciones espaciales y ambientales:

- Reconozco características de la Tierra que la hacen un planeta vivo.

- Utilizo coordenadas, convenciones y escalas para trabajar con mapas y planos de representación.

- Reconozco y utilizo los husos horarios.

- Localizo diversas culturas en el espacio geográfico y reconozco las principales características físicas de su entorno.

- Establezco relaciones entre la ubicación geoespacial y las características climáticas del entorno de diferentes culturas.

- Describo las características que permiten dividir a Colombia en regiones naturales (MEN, 2004).

Además de lo anterior se tuvieron en cuenta las cuatro primeras unidades del libro Los Caminos del Saber 6 (Santillana, 2013), a mencionar: Unidad 1. El universo; Unidad 2. La Tierra, nuestro planeta; Unidad 3. Geografía física del mundo y Unidad 4, Mesopotamia y Egipto, ya que son mucho más explícitas que los estándares, aunque con gran cantidad de contenido, por lo cual a la hora de hacer el diseño de la Aplicación se usaron esquemas que ayudaron a jerarquizar la información más importante de acuerdo al criterio de la docente investigadora.

Una propuestadesdeel Constructivismo

Teniendo en cuenta que el modelo pedagógico de la Institución Educativa es el Constructivismo desde el enfoque de la enseñanza para la comprensión, se menciona como una categoría imperiosa relacionada directamente con la forma en la que los y las estudiantes asimilaron los contenidos de la asignatura de Ciencias Sociales por medio de "Sociales Android", basándose en el Constructivismo Social de Vygotsky afirma: 
Constructivismo Social es aquel modelo basado en el constructivismo, que dicta que el conocimiento además de formarse a partir de las relaciones ambiente-yo, es la suma del factor entorno social a la ecuación: Los nuevos conocimientos se forman a partir de los propios esquemas de la persona producto de su realidad, y su comparación con los esquemas de los demás individuos que lo rodean (párr.7).

A este respecto durante el desarrollo del proyecto de investigación se pudo evidenciar que los estudiantes aprenden de manera diversa y que los modelos educativos actuales tienden a formarlos como si fuesen iguales, por lo cual desde el constructivismo se entiende que cada estudiante es un mundo diferente y que desde su entorno particular llega a las instituciones educativas con unos esquemas y códigos que muchas veces el sistema educativo no comprende, con la aplicación usada como estrategia pedagógica los niños y niñas pudieron explorar de manera autónoma e individual los contenidos de la asignatura de Ciencias Sociales, además de que por medio del trabajo libre pudieron compartir experiencias $\mathrm{y}$ aprender de sus pares.

Asimismo se toma en consideración el siguiente esquema que explica de manera concreta como los OVA pueden articularse directamente a los postulados del constructivismo como modelo de aprendizaje.

Figura 1 Aprendizaje constructivista

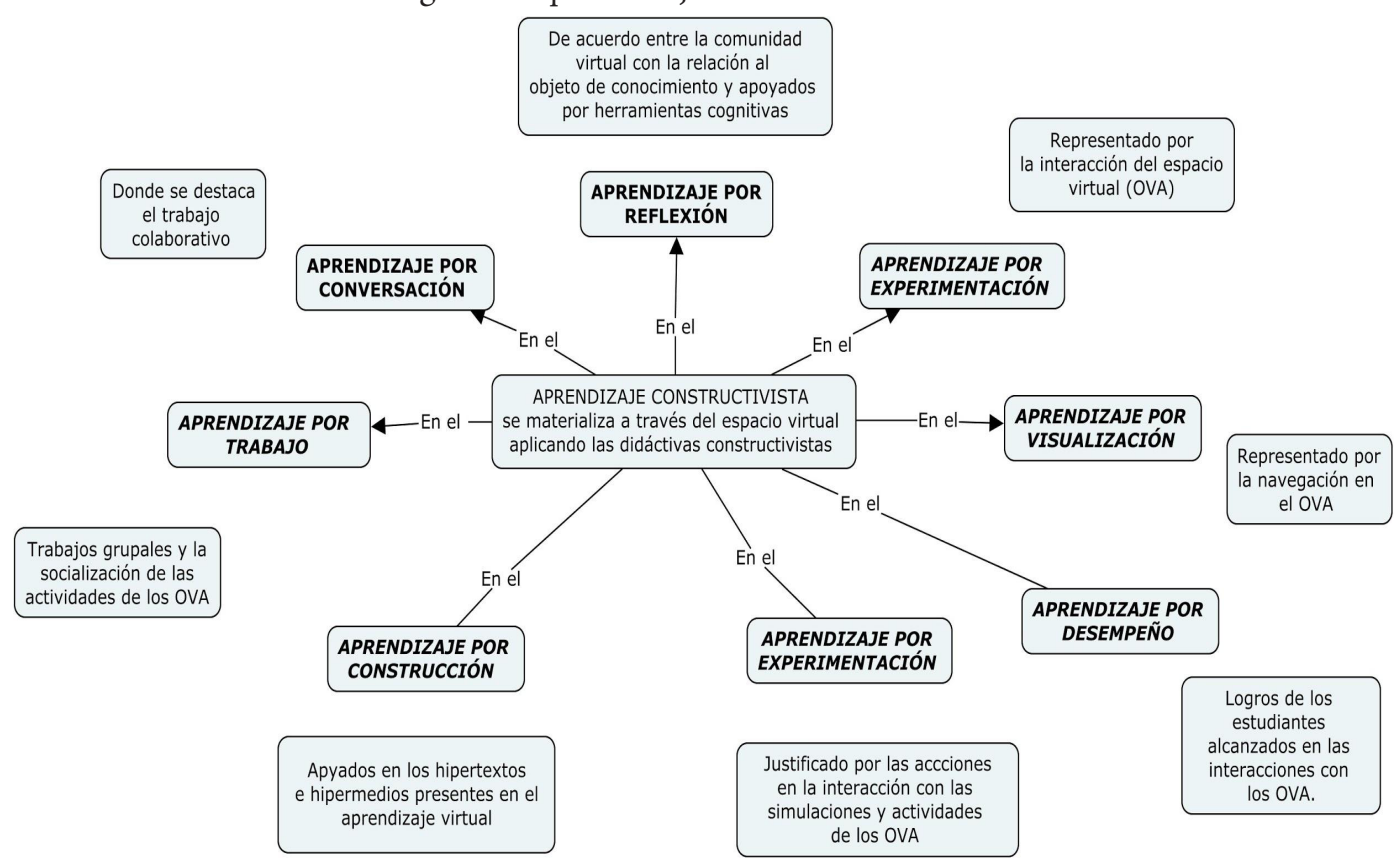

Fuente: Capacho, J. (2011) evaluación del Aprendizaje en Espacios Virtuales TIC. Barranquilla (Colombia): Editorial Universidad del Norte. P 114 
Aprendizaje significativo

Es pertinente mencionar respecto al manejo del tiempo con relación a los Estándares y los contenidos que en muchos docentes existe la preocupación e incluso la angustia de abordarlos de manera rápida a lo largo del año escolar; porque al final del año lectivo deben haberse visto todos los contenidos del libro de texto, como si fuera acumulativo o bancario, en muchos casos sin importar si estos constituyeron un aprendizaje significativo para los estudiantes, como dice Ausubel (1983):

Un aprendizaje es significativo cuando los contenidos: Son relacionados de modo no arbitrario y sustancial (no al pie de la letra) con lo que el alumno ya sabe. Por relación sustancial y no arbitraria se debe entender que las ideas se relacionan con algún aspecto existente específicamente relevante de la estructura cognoscitiva del alumno, como una imagen, un símbolo ya significativo, un concepto o una proposición (p.18).

En la presente investigación y gracias a que el proceso de evaluación fue constante, se pudo constatar por medio de los diarios de campo que los estudiantes construyeron conocimiento significativo de la asignatura porque con el transcurrir de las clases lo iban aplicando a la realidad que los rodeaba.

Análisis de los avances de la investigación

Teniendo en cuenta el objetivo general de la investigación que era evidenciar la aplicación de una estrategia pedagógica por medio de OVA, con el fin de transformar y enriquecer las prácticas docentes en las Ciencias Sociales, es pertinente mencionar que en el punto de culminación y evaluación de todo el proceso, esta investigación ha respondido satisfactoriamente, ya que se evidenció que si los docentes incorporan en sus metodologías el uso de herramientas tecnológicas por medio de la planeación responsable de Entornos Virtuales de Aprendizaje, todo el proceso en el aula de clase cambia de manera positiva tanto para el maestro porque ve que su esfuerzo y dedicación se ven recompensados con los resultados del trabajo participativo y activo de los estudiantes, y para los últimos porque cambian la perspectiva que tienen del sistema educativo.

Aunque el proceso investigativo no ha terminado y se encuentra en la etapa final en la que se pretende valorar el desempeño de los contenidos programáticos del área de ciencias sociales para el primer y segundo periodo con el uso del aplicativo móvil en los estudiantes de grado sexto por medio de la evaluación de los procesos cognitivos, procedimentales y actitudinales, es pertinente señalar que la evaluación se ha hecho tomando en cuenta su función pedagógica.

La función pedagógica tiene que ver directamente con la comprensión, regulación y mejora de la situación de enseñanza y aprendizaje. En este sentido se evalúa para obtener información que permita en un momento determinado saber qué pasó con el aprendizaje de 
los alumnos y con las respectivas estrategias de enseñanza asociadas a éste, para que en ambos casos, puedan hacerse las mejoras y ajustes necesarios (Díaz \& Rojas, 2010, p. 309).

De esta manera y para el caso en particular se evaluó el proceso desde sus inicios ya que la aplicación Android se realizó con dos de las cuatro unidades necesarias, con el fin de que los estudiantes la conocieran, la validaran y dieran a conocer su punto de vista sobre lo que les gustó y lo que les desagrado, con esta información se hicieron los cambios pertinentes para las dos últimas unidades, esto porque la idea era que los estudiantes fueran partícipes activos de su aprendizaje y que mejor que ellos mismos para evaluar los instrumentos y metodologías usados para su formación. En este proceso también se corrigieron errores tanto de forma como de fondo, y fue una construcción que permitió la comunicación asertiva y la participación activa tanto de la docente como de los estudiantes.

\section{Conclusiones}

El desarrollo de la investigación en el aula exige la necesidad de repensar el papel de los docentes y sus estrategias didácticas, para reenfocar la educación desde otras perspectivas, con nuevos recursos, con iniciativas propias, no necesariamente adquiridos a grandes costos, elaborados de forma participativa, teniendo en cuenta las necesidades del contexto y los conocimientos previos de los niños y niñas, con el fin de generar un aprendizaje significativo y desarrollar la dimensión afectiva y una mayor potencialidad en las aulas de clase.

La mediación tecnológica en el aula de clase, con Objetos virtuales de Aprendizaje OVA, requiere sistematizar las experiencias con el fin de redireccionar el aprendizaje de una manera significativa en cuanto a las posibilidades de enseñanza, en las que se debe incluir a los estudiantes. Por otra parte es un incentivo a los docentes en general para que se atrevan a sobrepasar el uso exclusivo de libros de texto y la clase magistral y puedan plasmar en un OVA muchas de las ideas que tienen en mente pero que nunca se materializan.

Asimismo se deja abierta la posibilidad para que las Instituciones Educativas creen Entornos Virtuales de Aprendizaje de manera interdisciplinar y los docentes puedan diseñar sus clases desde Objetos Virtuales de Aprendizaje que tengan en cuenta las necesidades y las posibilidades de aprendizaje de sus estudiantes, pero de manera conjunta y cooperativa para priorizar el trabajo colectivo, la interdisciplinariedad $\mathrm{y}$ la resolución de problemas, pues es erróneo pensar que el constructivismo solo se aplica para los estudiantes, si se hiciera entre maestros se engrandecería la labor docente.

También es importante estimular la investigación docente, porque en las aulas se presentan los problemas de la didáctica y las posibilidades para superarlos con creatividad; pero lastimosamente eso solo se queda en la buena experiencia, ya que nada 
se sistematiza, la investigación da de enseñanza y aprendizaje en las esa posibilidad junto con varias Instituciones Educativas fuera más herramientas que harían que el proceso favorable y pudiera fortalecerse.

\section{Referentes}

Ausubel, D. P., Novak, J., \& Hanesian, H. ((2009)). Psicología Educativa: Un punto de vista cognoscitivo. México: $2^{\circ}$ Ed. Trillas.

Belloch, C. (s.f.). Entornos Virtuales de Aprendizaje. 2.

Botero, J. (2014). Propuesta de un objeto virtual de aprendizaje (OVA) para la enseñanzaaprendizaje de la cinética química (Tesis de maestría). Bogotá: Universidad Nacional de Colombia.

Capacho, J. (2011). Evaluación del Aprendizaje en Espacios Virtuales TIC. Barranquilla (Colombia): Editorial Universidad del Norte.

COLOMBIA Ministerio de Educación Nacional (2002) Estándares Básicos de Competencias en ciencias sociales. Bogotá MEN

Díaz, F., \& Hernández, G. (2010). Estrategias docentes para un aprendizaje significativo una Interpretación Constructivista. México: McGraw Hill.

Elliott, J. (2000). La investigación-acción en educación. Madrid: Morata.

Elliot, J. (2005). El cambio educativo desde la educación acción. Madrid: Morata.

Pulido, D., Nájar, O., \& Guesguan, L. (2016). Vivamos la innovación de la inclusión de dispositivos móviles en la educación. Praxis \& Saber, 7(14), 115 - 140. https://doi.org/10.19053/22160159.5220

Sampieri, H., Collado, F., \& Lucio, M. (2014). Metodología de la investigación. México: McGraw-Hill.

Santillana. (2013). Los Caminos del Saber 6. Bogotá: Santillana.

Serres, M. (2014). Pulgarcita. Barcelona: Gedisa.

Zemelman, H. (2017). Pensar teórico y pensar epistémico: los retos de las ciencias sociales latinoamericanas. México D.C., México. 